Research Article

\title{
Inequalities Involving Essential Norm Estimates of Product-Type Operators
}

\author{
Manisha Devi $\mathbb{D}^{\mathrm{D}}$, Ajay K. Sharma, and Kuldip Raj \\ School of Mathematics, Shri Mata Vaishno Devi University, Katra 182320, Jammu and Kashmir, India \\ Correspondence should be addressed to Kuldip Raj; kuldipraj68@gmail.com
}

Received 11 September 2020; Revised 22 January 2021; Accepted 15 February 2021; Published 3 March 2021

Academic Editor: Ming-Sheng Liu

Copyright ( 92021 Manisha Devi et al. This is an open access article distributed under the Creative Commons Attribution License, which permits unrestricted use, distribution, and reproduction in any medium, provided the original work is properly cited.

Consider an open unit disk $\mathbb{D}=\{z \in \mathbb{C}:|z|<1\}$ in the complex plane $\mathbb{C}, \xi$ a holomorphic function on $\mathbb{D}$, and $\psi$ a holomorphic selfmap of $\mathbb{D}$. For an analytic function $f$, the weighted composition operator is denoted and defined as follows: $\left(\mathscr{W}_{\xi, \psi} f\right)(z)=\xi(z) f(\psi(z))$. We estimate the essential norm of this operator from Dirichlet-type spaces to Bers-type spaces and Bloch-type spaces.

\section{Introduction and Preliminaries}

Consider an open unit disk $\mathbb{D}=\{z \in \mathbb{C}:|z|<1\}$ in the complex plane $\mathbb{C}$. Let $H(\mathbb{D})$ denote the class of all analytic functions on $\mathbb{D}, S(\mathbb{D})$ be the class of all holomorphic selfmaps of $\mathbb{D}$, and $H^{\infty}$ be the space of all bounded holomorphic functions on $\mathbb{D}$. Let $\xi \in H(\mathbb{D})$ and $\psi$ be a holomorphic selfmap of $\mathbb{D}$. For $z \in \mathbb{D}$, the composition operator and multiplication operator are, respectively, defined by

$$
\begin{array}{r}
\left(C_{\psi} f\right)(z)=f(\psi(z)), \\
\left(M_{\xi} f\right)(z)=\xi(z) f(z), \\
\\
f \in H(\mathbb{D}) .
\end{array}
$$

The weighted composition operator is denoted and defined as

$$
\left(\mathscr{W}_{\xi, \psi} f\right)(z)=\xi(z) f(\psi(z)), \quad f \in H(\mathbb{D}),
$$

where $\mathscr{W}_{\xi, \psi}$ is a product-type operator as $\mathscr{W}_{\xi, \psi}=M_{\xi} C_{\psi}$. Clearly, this operator can be seen as a generalization of the composition operator and multiplication operator. It can be easily seen that, for $\xi \equiv 1$, the operator reduced to $C_{\psi}$. If $\psi(z)=z$, the operator gets reduced to $M_{\xi}$. This operator is basically a linear transformation of $H(\mathbb{D})$ defined by $\left(\mathscr{W}_{\xi, \psi} f\right)(z)=\xi(z) f(\psi(z))=\left(M_{\xi} C_{\psi} f\right)(z)$, for $f$ in $H(\mathbb{D})$ and $z$ in $\mathbb{D}$. The basic aim is to give the operator-theoretic characterization of these operators in terms of functiontheoretic characterization of their including functions. Various holomorphic function spaces on various domains have been studied for the boundedness and compactness of weighted composition operators acting on them. Moreover, a number of papers have been studied on these operators acting on different spaces of holomorphic functions on various domains. For more details, see [1-14] and the references therein. We say that a linear operator is bounded if the image of a bounded set is a bounded set. Moreover, a linear operator is said to be compact if it maps the bounded sets to those sets whose closure is compact. For each $\alpha>0$, the weighted Bloch space $\mathfrak{B}$ is defined as follows:

$$
\mathfrak{B}=\left\{f \in H(\mathbb{D}): \sup _{z \in \mathbb{D}}\left(1-|z|^{2}\right)^{\alpha}\left|f^{\prime}(z)\right|<\infty\right\} .
$$

In this expression, seminormed is defined. This space forms a Banach space with the natural norm defined by

$$
\|f\|_{\mathfrak{B}}=|f(0)|+\sup _{z \in \mathbb{D}}\left(1-|z|^{2}\right)^{\alpha}\left|f^{\prime}(z)\right| \text {. }
$$

For $\alpha=1$, this space gets reduced to classical Bloch space. A function $\omega: \mathbb{D} \longrightarrow(0, \infty)$ is said to be a weight if it is continuous. For $z \in \mathbb{D}$, the weight $\omega$ is said to be radial if $\omega(z)=\omega(|z|)$. A weight $\omega$ is said to be a standard weight if 
$\lim _{|z| \longrightarrow 1^{-1}} \omega(z)=0$. For a weight function $\omega$, the Bloch-type space $\mathfrak{B}_{\omega}$ is defined by

$$
\mathfrak{B}_{\omega}=\left\{f \in H(\mathbb{D}): \sup _{z \in \mathbb{D}} \omega(z)\left|f^{\prime}(z)\right|<\infty\right\} .
$$

The little Bloch-type space $\mathfrak{B}_{\omega, 0}$ is the closure of the set of polynomials in $\mathfrak{B}_{\omega}$ and is defined as follows:

$$
\mathfrak{B}_{\omega, 0}=\left\{f \in \mathfrak{B}_{\omega}: \lim _{|z| \longrightarrow 1} \omega(z)\left|f^{\prime}(z)\right|=0\right\} .
$$

Both $\mathfrak{B}_{\omega}$ and $\mathfrak{B}_{\omega, 0}$ form a Banach space with the following norm:

$$
\|f\|_{\mathfrak{B}_{\omega}}=|f(0)|+\sup _{z \in \mathbb{D}} \omega(z)\left|f^{\prime}(z)\right| .
$$

For more information about these spaces, one may refer $[1-3,5,6,15,16]$ and the references therein. Likewise, for weight $\omega$, the Bers-type space $\mathfrak{A}_{\omega}$ is defined as follows:

$$
\mathfrak{\mathcal { A }}_{\omega}=\left\{f \in H(\mathbb{D}):\|f\|_{\mathfrak{A}_{\omega}}:=\sup _{z \in \mathbb{D}} \omega(z)|f(z)|<\infty\right\} .
$$

It is a nonseparable Banach space with the norm $\|\cdot\|_{\mathfrak{A}}$. The closure of the set of polynomials in $\mathfrak{A}_{\omega}$ forms a separable Banach space. This set is denoted by $\mathfrak{A}_{\omega, 0}$ and is defined as

$$
\mathfrak{\mathfrak { A }}_{\omega, 0}=\left\{f \in \mathfrak{\mathfrak { A }}_{\omega}: \lim _{|z| \longrightarrow 1^{-}} \omega(z)|f(z)|=0\right\} .
$$

These spaces and their properties are discussed in many papers; some of these are $[3,15,16]$ and the references therein. The Dirichlet space is defined as follows:

$$
\mathfrak{D}=\left\{f \in H(\mathbb{D}): \int_{\mathbb{D}}\left|f^{\prime}(z)\right|^{2} \mathrm{~d} A(z)<\infty\right\},
$$

where $\mathrm{d} A(z)$ denotes the normalized Lebesgue area measure on $\mathbb{D}$. With the following norm, it is a Hilbert space:

$$
\|f\|_{\mathfrak{D}}^{2}=|f(0)|^{2}+\int_{\mathbb{D}}\left|f^{\prime}(z)\right|^{2} \mathrm{~d} A(z) .
$$

Consider a function $K:[0, \infty) \longrightarrow[0, \infty)$ which is right continuous and increasing. In this paper, we consider function $K$ as a weight function. With a weight function $K$, the Dirichlet type space $\mathfrak{D}_{K}$ is given as follows:

$$
\mathfrak{D}_{K}=\left\{f \in H(\mathbb{D}): \int_{\mathbb{D}}\left|f^{\prime}(z)\right|^{2} K\left(1-|z|^{2}\right) \mathrm{d} A(z)<\infty\right\} .
$$

Clearly, space $\mathfrak{D}_{K}$ forms a Hilbert space with the norm $\|\cdot\|_{\mathfrak{D}_{K}}$ defined by

$$
\|f\|_{\mathfrak{D}_{K}}^{2}=|f(0)|^{2}+\int_{\mathbb{D}}\left|f^{\prime}(z)\right|^{2} K\left(1-|z|^{2}\right) \mathrm{d} A(z) .
$$

Here, we have $K(t)=t^{p}, 0 \leq p<\infty$, and $\mathfrak{D}_{K}$ gives $\mathfrak{D}_{p}$, that is, the usual Dirichlet-type space. This gives a classical Dirichlet space $\mathfrak{D}$ for a case when $p=0$ and for $p=1$, and we gain the Hardy space $H^{2}$. These spaces have been studied widely in various papers. For example, Aleman, in [17], obtained that each element of $\mathfrak{D}_{K}$ can be written as a quotient of two bounded functions in $\mathfrak{D}_{K}$. Kerman and Sawyer [18], by taking some conditions on weight function $K$, characterized Carleson measures and multipliers of $\mathfrak{D}_{K}$ in terms of maximal operator.

The Möbius invariant space generated by $\mathfrak{D}_{K}$ is denoted by $Q_{K}$. The space $\mathbb{Q}_{K}$ contains those functions $f \in H(\mathbb{D})$ which satisfy the following:

$$
\sup _{a \in \mathbb{D}}\left\|f^{\circ} \sigma_{\mathrm{a}}-\mathrm{f}(\mathrm{a})\right\|_{\mathfrak{D}_{K}}<\infty,
$$

where $\sigma_{a}(z)=((a-z) /(1-\bar{a} z))$ is the Möbius transformation of $\mathbb{D}$. Wulan and Zhu, in [19], characterized Lacunary series in the $\mathbb{Q}_{K}$ space under some conditions on weight function $K$. Furthermore, Wulan and Zhou [20] characterized space $Q_{K}$ in terms of fractional-order derivatives of function. They also established a relationship between Morrey type spaces and $Q_{K}$ space in terms of fractional order derivatives. In the study of $Q_{K}$ spaces, the following two conditions play a very important role:

$$
\begin{aligned}
& \int_{0}^{1} \frac{\varphi_{K}(s)}{s} \mathrm{~d} s<\infty, \\
& \int_{1}^{\infty} \frac{\varphi_{K}(s)}{s^{2}} \mathrm{~d} s<\infty,
\end{aligned}
$$

where

$$
\varphi_{K}(s)=\sup _{0 \leq t \leq 1} \frac{K(s t)}{K(t)}, \quad 0<s<\infty .
$$

Let $M\left(\mathfrak{D}_{K}\right)$ be the class of multipliers of $\mathfrak{D}_{K}$, that is,

$$
M\left(\mathfrak{D}_{K}\right)=\left\{f \in H(\mathbb{D}): f g \in \mathfrak{D}_{K}, \forall g \in \mathfrak{D}_{K}\right\} .
$$

Bao et al., in [21], characterized the interpolating sequences for $M\left(\mathfrak{D}_{K}\right)$ of space $\mathfrak{D}_{K}$, under certain conditions of weight function $K$. They also obtained corona theorem, $\bar{\partial}$ - equation, and corona-type decomposition theorem on $M\left(\mathfrak{D}_{K}\right)$. For more details, see $[9,15,21-25]$ and the references therein.

From [26], one can see that if $K$ satisfies (1), then

$$
K_{1}(t)=\int_{0}^{t} K(s) \frac{\mathrm{d} s}{s} \approx K(t), \quad 0<t<1 .
$$

If $K$ satisfies (16), then

$$
K_{2}(t)=t \int_{t}^{\infty} K(s) \frac{\mathrm{d} s}{s^{2}} \approx K(t), \quad t>0
$$

From condition (16), we get that $K(2 t) \approx K(t)$ for $0<t<1$. Also, there exist $C>0$ sufficiently small for which $t^{-C} K_{1}(t)$ is increasing and $K_{2}(t) t^{C-1}$ is decreasing. For more information about weight function $K$, one can refer [19-21].

The criterion of boundedness as well as compactness has been discussed in many papers. Recently, Gürbüz, in [27], studied the boundedness of generalized commutators of rough fractional maximal and integral operators on generalized weighted Morrey spaces, respectively, and, in [28], he investigated the generalized weighted Morrey estimates for the boundedness of Marcinkiewicz integrals with rough kernel associated with Schrödinger operator and their 
commutators. Furthermore, in [29], Gürbuüz studied the behavior of multi-sublinear fractional maximal operators and rough multilinear fractional integral both on product $L^{p}$ and weighted $L^{p}$ spaces and, in [30], he obtained the boundedness of the variation and oscillation operators for the family of multilinear integrals with Lipschitz functions on weighted Morrey spaces. Among others in [3], we obtained the following results about boundedness and compactness of $\mathscr{W}_{\xi, \psi}$ given as follows.

Theorem 1. Let $\omega$ and $K$ be two weight functions, $\xi \in H(\mathbb{D})$, and $\psi$ be a self-holomorphic map on $\mathbb{D}$. Then, the operator $\mathscr{W}_{\xi, \psi}: \mathfrak{D}_{K} \longrightarrow \mathfrak{B}_{\omega}$ is bounded if and only if the following conditions hold:

(i) $M_{1}=\sup _{z \in \mathbb{D}}\left(\left(\omega(z)\left|\xi^{\prime}(z)\right|\right) /\left(\sqrt{K\left(1-|\psi(z)|^{2}\right)}(1-\right.\right.$ $\left.\left.\left.|\psi(z)|^{2}\right)\right)\right)<\infty$

(ii) $M_{2}=\sup _{z \in \mathbb{D}}\left(\left(\omega(z)\left|\xi(z) \psi^{\prime}(z)\right|\right) /\left(\sqrt{K\left(1-|\psi(z)|^{2}\right)}\right.\right.$ $\left.\left.\left(1-|\psi(z)|^{2}\right)^{2}\right)\right)<\infty$

Furthermore, if the operator $\mathscr{W}_{\xi, \psi}: \mathfrak{D}_{K} \longrightarrow \mathfrak{B}_{\omega}$ is bounded, then

$$
M_{1}+M_{2} \leq\left\|\mathscr{W}_{\xi, \psi}\right\|_{\mathfrak{D}_{K} \longrightarrow \mathfrak{B}_{\omega}} \lesssim 1+M_{1}+M_{2} .
$$

Theorem 2. Let $\omega$ be a standard weight, $\xi \in H(\mathbb{D})$, and $\psi$ be a self-holomorphic map on $\mathbb{D}$. Let $K$ be a weight function. Assume that $\mathscr{W}_{\xi, \psi}: \mathfrak{D}_{K} \longrightarrow \mathfrak{B}_{\omega}$ is bounded. Then, the operator $\mathscr{W}_{\xi, \psi}: \mathfrak{D}_{K} \longrightarrow \mathfrak{B}_{\omega}$ is compact if and only if the following conditions hold:

$$
\|T\|_{e, X_{1} \longrightarrow X_{2}}=\inf \left\{\|T-E\|_{X_{1} \longrightarrow X_{2}}: E \text { mapping } X_{1} \text { into } X_{2} \text { is compact }\right\}
$$

where $\|\cdot\|_{X_{1} \longrightarrow X_{2}}$ is the operator norm. In other words, the essential norm is the distance from compact operators $E$ mapping $X_{1}$ into $X_{2}$ to the bounded linear operator $T: X_{1} \longrightarrow X_{2}$. If $X_{1}=X_{2}$, that is, the two Banach spaces are same, then the norm is simply denoted by $\|\cdot\|_{e}$. For unbounded linear operator $T: X_{1} \longrightarrow X_{2}$, we have $\|T\|_{e, X_{1} \longrightarrow X_{2}}=\infty$. As the class of all compact operators is contained in the class of all bounded operators, in fact, this subset is closed, which implies that the operator $T$ is compact if and only if $\|T\|_{e, X_{1}} \longrightarrow X_{2}=0$. Thus, the estimate of essential norm leads to the compactness of the operator. Various results on the essential norm of different operators such as multiplication, composition, differentiation, weighted composition, generalized weighted composition, and their different combinations are studied in numerous research papers, and some of the references are [31-37].

This study is formulated in a systematic way. Introduction and literature part is kept in Section 1. In Section 2, we estimated the essential norm of operator $\mathscr{W}_{\xi, \psi}: \mathfrak{D}_{K} \longrightarrow \mathfrak{B}_{\omega}$. Finally, in Section 3, we estimated the essential norm of operator $\mathscr{W}_{\xi, \psi}: \mathfrak{D}_{K} \longrightarrow \mathfrak{A}_{\omega}$. Throughout the paper, the notation $a \leq b$, for any two positive quantities $a$ and $b$, which means that $a \leq C b$, where $C$ is some positive

$$
\begin{aligned}
& \text { (i) } \lim _{|\psi(z)| \longrightarrow 1}\left(\left(\omega(z)\left|\xi^{\prime}(z)\right|\right) /\left(\sqrt{K\left(1-|\psi(z)|^{2}\right)}(1-\right.\right. \\
& \left.\left.\left.|\psi(z)|^{2}\right)\right)\right)=0 \\
& \text { (ii) } \lim _{|\psi(z)| \longrightarrow 1}\left(\left(\omega(z)\left|\xi(z) \psi^{\prime}(z)\right|\right) /\left(\sqrt{K\left(1-|\psi(z)|^{2}\right)}\right.\right. \\
& \left.\left.\quad\left(1-|\psi(z)|^{2}\right)^{2}\right)\right)=0
\end{aligned}
$$

Theorem 3. Let $\omega$ be a weight and $K$ be a weight function, $\xi \in H(\mathbb{D})$, and $\psi$ be a self-holomorphic map on $\mathbb{D}$. Then, the operator $\mathscr{W}_{\xi, \psi}: \mathfrak{D}_{K} \longrightarrow \mathfrak{\mathfrak { A }}_{\omega}$ is bounded if and only if the following condition holds:

$$
l_{1}=\sup _{z \in \mathbb{D}} \frac{\omega(z)|\xi(z)|}{\sqrt{K\left(1-|\psi(z)|^{2}\right)}\left(1-|\psi(z)|^{2}\right)}<\infty .
$$

Theorem 4. Let $\omega$ be a standard weight, $\xi \in H(\mathbb{D})$, and $\psi$ be a self-analytic map on $\mathbb{D}$. Let $K$ be a weight function. Assume that the operator $\mathscr{W}_{\xi, \psi}: \mathfrak{D}_{K} \longrightarrow \mathfrak{A}_{\omega}$ is bounded. Then, $\mathscr{W}_{\xi, \psi}: \mathfrak{D}_{K} \longrightarrow \mathfrak{A}_{\omega}$ is compact if and only if the following condition is satisfied:

$$
\lim _{|\psi(z)| \longrightarrow 1^{-}} \frac{\omega(z)|\xi(z)|}{\sqrt{K\left(1-|\psi(z)|^{2}\right)}\left(1-|\psi(z)|^{2}\right)}=0 .
$$

The aim of this paper is to provide some estimates of essential norm of the operator $\mathscr{W}_{\xi, \psi}: \mathfrak{D}_{K} \longrightarrow \mathfrak{B}_{\omega}$ as well as of $\mathscr{W}_{\xi, \psi}: \mathfrak{D}_{K} \longrightarrow \mathfrak{A}_{\omega}$.

Assume that $T: X_{1} \longrightarrow X_{2}$ is a bounded linear operator for Banach spaces $X_{1}$ and $X_{2}$. The essential norm of operator $T$ is denoted and defined as follows:

constant. The value of constant $C$ may change from one place to the other. We write $a \approx b$ if $a \lesssim b$ and $b \lesssim a$.

\section{Essential Norm of Weighted Composition Operator from Dirichlet-Type Space to Bloch-Type Space}

Theorem 5. Let $\omega$ be a standard weight, $\xi \in H(\mathbb{D})$, and $\psi$ be a self-analytic map on $\mathbb{D}$. Let $K$ be a weight function. Assume that $\mathscr{W}_{\xi, \psi}: \mathfrak{D}_{K} \longrightarrow \mathfrak{B}_{\omega}$ is bounded. Then,

$$
\left\|\mathscr{W}_{\xi, \psi}\right\|_{e, \mathfrak{D}_{K} \longrightarrow \mathfrak{B}_{\omega}} \approx \max \{R, S\}
$$

where

$$
\begin{aligned}
& R:=\limsup _{|\psi(z)| \longrightarrow 1} \frac{\omega(z)\left|\xi^{\prime}(z)\right|}{\sqrt{K\left(1-|\psi(z)|^{2}\right)}\left(1-|\psi(z)|^{2}\right)} \\
& S:=\limsup _{|\psi(z)| \longrightarrow 1} \frac{\omega(z)\left|\xi(z) \psi^{\prime}(z)\right|}{\sqrt{K\left(1-|\psi(z)|^{2}\right)}\left(1-|\psi(z)|^{2}\right)^{2}}
\end{aligned}
$$


Proof. At first, we show that

$$
\left\|\mathscr{W}_{\xi, \psi}\right\|_{e, \mathfrak{D}_{K} \longrightarrow \mathfrak{B}_{\omega}} \geq \max \{R, S\} .
$$

For $z \in \mathbb{D}$, define a function

$$
\begin{aligned}
h_{z}(w)= & a_{0} \frac{\left(1-|z|^{2}\right)^{\varepsilon / 2}}{\sqrt{K\left(1-|z|^{2}\right)}(1-\bar{z} w)^{1+(\varepsilon / 2)}} \\
& +a_{1} \frac{\left(1-|z|^{2}\right)^{1+(\varepsilon / 2)}}{\sqrt{K\left(1-|z|^{2}\right)}(1-\bar{z} w)^{2+(\varepsilon / 2)}},
\end{aligned}
$$

where $a_{0}=(2+\varepsilon / 2)$ and $a_{1}=-(1+\varepsilon / 2)$. It can be easily checked that $h_{z} \in \mathbb{D}_{K}$ and, for all $z \in \mathbb{D},\left\|h_{z}\right\|_{\mathfrak{D}_{K}} \lesssim 1$. On calculation, we have $h_{z}^{\prime}(z)=0$ and $h_{z}(z)=1 /\left(\sqrt{K\left(1-|z|^{2}\right)}\left(1-|z|^{2}\right)\right)$. Furthermore, on compact subsets of $\mathbb{D}, h_{z}$ converges to zero as $|z| \longrightarrow 1$. Hence, for any compact operator $E$ : $\mathfrak{D}_{K} \longrightarrow \mathfrak{B}_{\omega}$ and any $\left(\zeta_{n}\right)_{n \in \mathbb{D}}$ such that $\left|\psi\left(\zeta_{n}\right)\right| \longrightarrow 1$, we obtain

$$
\begin{aligned}
\left\|\mathscr{W}_{\xi, \psi}-E\right\|_{\mathfrak{D}_{K} \longrightarrow \mathfrak{B}_{\omega}} & \geq\left\|\left(\mathscr{W}_{\xi, \psi}-E\right) h_{\psi\left(\zeta_{n}\right)}\right\|_{\mathfrak{B}_{\omega}} \\
& \geq\left\|\mathscr{W}_{\xi, \psi} h_{\psi}\left(\zeta_{n}\right)\right\|_{\mathfrak{B}_{\omega}}-\left\|E h_{\psi\left(\zeta_{n}\right)}\right\|_{\mathfrak{B}_{\omega}} .
\end{aligned}
$$

In the above inequality, take $\lim \sup _{\left|\psi\left(\zeta_{n}\right)\right| \longrightarrow 1}$ on both sides, and we obtain

$$
\left\|\mathscr{W}_{\xi, \psi}-E\right\|_{\mathfrak{D}_{K} \longrightarrow \mathfrak{B}_{\omega}} \gtrsim \limsup _{\left|\psi\left(\zeta_{n}\right)\right| \longrightarrow 1}\left\|\mathscr{W}_{\xi, \psi} h_{\psi}\left(\zeta_{n}\right)\right\|_{\mathfrak{B}_{\omega}} \gtrsim \limsup _{\left|\psi\left(\zeta_{n}\right)\right| \longrightarrow 1} \frac{\omega\left(\zeta_{n}\right)\left|\xi^{\prime}\left(\zeta_{n}\right)\right|}{\sqrt{K\left(1-\left|\psi\left(\zeta_{n}\right)\right|^{2}\right)}\left(1-\left|\psi\left(\zeta_{n}\right)\right|^{2}\right)}=R .
$$

Again, for $z \in \mathbb{D}$, define another function:

$$
\begin{aligned}
k_{z}(w)= & b_{0} \frac{\left(1-|z|^{2}\right)^{\varepsilon / 2}}{\sqrt{K\left(1-|z|^{2}\right)}(1-\bar{z} w)^{1+\varepsilon / 2}} \\
& +b_{1} \frac{\left(1-|z|^{2}\right)^{1+\varepsilon / 2}}{\sqrt{K\left(1-|z|^{2}\right)}(1-\bar{z} w)^{2+\varepsilon / 2}}
\end{aligned}
$$

where $b_{0}=1$ and $b_{1}=-1$. In the similar manner, we can check that $k_{z} \in \mathbb{D}_{K}$ and, for all $z \in \mathbb{D},\left\|k_{z}\right\|_{\mathfrak{D}_{K}} \lesssim 1$. On calculation, we have $k_{z}(z)=0$ and $k_{z}^{\prime}(z)=-\bar{z} /\left(\sqrt{K\left(1-|z|^{2}\right)}\left(1-|z|^{2}\right)^{2}\right)$. Furthermore, on compact subsets of $\mathbb{D}, k_{z}$ converges to zero as $|z| \longrightarrow 1$. Thus, for any compact operator $E$ : $\mathfrak{D}_{K} \longrightarrow \mathfrak{B}_{\omega}$ and any $\left(\zeta_{n}\right)_{n \in \mathbb{D}}$ such that $\left|\psi\left(\zeta_{n}\right)\right| \longrightarrow 1$, we obtain

$$
\begin{aligned}
\left\|\mathscr{W}_{\xi, \psi}-E\right\|_{\mathfrak{D}_{K} \longrightarrow \mathfrak{B}_{\omega}} \geq & \left\|\left(\mathscr{W}_{\xi, \psi}-E\right) k_{\psi\left(\zeta_{n}\right)}\right\|_{\mathfrak{B}_{\omega}} \geq\left\|\mathscr{W}_{\xi, \psi} k_{\psi\left(\zeta_{n}\right)}\right\|_{\mathfrak{B}_{\omega}} \\
& -\left\|E k_{\psi\left(\zeta_{n}\right)}\right\|_{\mathfrak{B}_{\omega}} \cdot
\end{aligned}
$$

By taking $\lim \sup _{\left|\psi\left(\zeta_{n}\right)\right| \longrightarrow 1}$ on both sides of the above inequality, we obtain

$$
\left\|\mathscr{W}_{\xi, \psi}-E\right\|_{\mathfrak{D}_{K} \longrightarrow \mathfrak{B}_{\omega}} \gtrsim \limsup _{\left|\psi\left(\zeta_{n}\right)\right| \longrightarrow 1}\left\|\mathscr{W}_{\xi, \psi} k_{\psi}\left(\zeta_{n}\right)\right\|_{\mathfrak{B}_{\omega}} \gtrsim \limsup _{\left|\psi\left(\zeta_{n}\right)\right| \longrightarrow 1} \frac{\omega\left(\zeta_{n}\right)\left|\xi\left(\zeta_{n}\right) \psi^{\prime}\left(\zeta_{n}\right)\right|}{\sqrt{K\left(1-\left|\psi\left(\zeta_{n}\right)\right|^{2}\right)}\left(1-\left|\psi\left(\zeta_{n}\right)\right|^{2}\right)^{2}}=S .
$$
that

On applying the definition of essential norm, we find

$$
\left\|\mathscr{W}_{\xi, \psi}\right\|_{e, \mathfrak{D}_{K} \longrightarrow \mathfrak{B}_{\omega}}=\inf _{E}\left\|\mathscr{W}_{\xi, \psi}-E\right\|_{\mathfrak{D}_{K} \longrightarrow \mathfrak{B}_{\omega}} \geq \max \{R, S\}
$$

Next, we prove that

$$
\left\|\mathscr{W}_{\xi, \psi}\right\|_{e, \mathfrak{D}_{K} \longrightarrow \mathfrak{B}_{\omega}} \lesssim \max \{R, S\} .
$$

For $\delta \in[0,1)$, consider $E_{\delta}: H(\mathbb{D}) \longrightarrow H(\mathbb{D})$, defined as follows:

$$
\left(E_{\delta} f\right)(z)=f_{\delta}(z)=f(\delta z), \quad f \in H(\mathbb{D}) .
$$

Clearly, $E_{\delta}$ is compact on $\mathfrak{D}_{K}$ and $\left\|E_{\delta}\right\|_{\mathfrak{D}_{K}} \longrightarrow \mathfrak{D}_{K} \leq 1$. Consider a sequence $\left\{\delta_{n}\right\} \subset(0,1)$ satisfying $\delta_{n} \longrightarrow 1$ as $n \longrightarrow \infty$. Then, for all $n \in \mathbb{N}$, operator $\mathscr{W}_{\xi, \psi} E_{\delta_{n}}: \mathfrak{D}_{K} \longrightarrow \mathfrak{B}_{\omega}$ is compact. By using the definition of essential norm, we obtain

$$
\left\|\mathscr{W}_{\xi, \psi}\right\|_{e, \mathfrak{D}_{K} \longrightarrow \mathfrak{B}_{\omega}} \leq \limsup _{n \longrightarrow \infty}\left\|\mathscr{W}_{\xi, \psi}-\mathscr{W}_{\xi, \psi} E_{\delta_{n}}\right\|_{\mathfrak{D}_{K} \longrightarrow \mathfrak{B}_{\omega}}
$$

Therefore, we only have to prove that

$$
\limsup _{n \longrightarrow \infty}\left\|\mathscr{W}_{\xi, \psi}-\mathscr{W}_{\xi, \psi} E_{\delta_{n}}\right\|_{\mathfrak{D}_{K} \longrightarrow \mathfrak{B}_{\omega}} \leqslant \max \{R, S\} .
$$
have

Let $f$ be a function in $\mathfrak{D}_{K}$ satisfying $\|f\|_{\mathfrak{D}_{K}} \leq 1$; then, we 


$$
\begin{aligned}
\left\|\left(\mathscr{W}_{\xi, \psi}-\mathscr{W}_{\xi, \psi} E_{\delta_{n}}\right) f\right\|_{\mathfrak{D}_{K} \rightarrow \mathfrak{B}_{\omega}}= & \left|\xi(0) f(\psi(0))-\xi(0) f\left(\delta_{n} \psi(0)\right)\right| \\
& +\left\|\xi\left(f-f_{\delta_{n}}\right)^{\circ} \psi\right\|_{\mathfrak{B}_{\omega}} .
\end{aligned}
$$

Clearly, $\quad \lim _{n \longrightarrow \infty}\left|\xi(0) f(\psi(0))-\xi(0) f\left(\delta_{n} \psi(0)\right)\right|=0$. Furthermore, consider a large enough $N \in \mathbb{N}$ such that, for all $n \geq N$, we have $\delta_{n} \geq 1 / 2$. Thus, we obtain

$$
\begin{aligned}
\limsup _{n \longrightarrow \infty}\left\|\xi\left(f-f_{\delta_{n}}\right) \circ \psi\right\|_{\mathfrak{B}_{\omega}} \leq & l \limsup _{n \longrightarrow \infty} \sup _{|\psi(z)| \leq \delta_{N}} \omega(z)\left|\xi(z)\left\|\left(f-f_{\delta_{n}}\right)^{\prime}(\psi(z))\right\| \psi^{\prime}(z)\right| \\
& +\limsup _{n \longrightarrow \infty} \sup _{|\psi(z)| \leq \delta_{N}} \omega(z)\left|\xi(z)\left\|\left(f-f_{\delta_{n}}\right)^{\prime}(\psi(z))\right\| \psi^{\prime}(z)\right| \\
& +\limsup _{n \longrightarrow \infty} \sup _{|\psi(z)| \leq \delta_{N}} \omega(z)\left|\xi^{\prime}(z) \|\left(f-f_{\delta_{n}}\right)(\psi(z))\right| \\
& +\limsup _{n \longrightarrow \infty} \sup _{|\psi(z)| \leq \delta_{N}} \omega(z)\left|\xi^{\prime}(z) \|\left(f-f_{\delta_{n}}\right)(\psi(z))\right| \\
= & S_{1}+S_{2}+S_{3}+S_{4},
\end{aligned}
$$

where

$$
\begin{aligned}
& S_{1}=\limsup _{n \longrightarrow \infty} \sup _{|\psi(z)| \leq \delta_{N}} \omega(z)\left|\xi(z)\left\|\left(f-f_{\delta_{n}}\right)^{\prime}(\psi(z))\right\| \psi^{\prime}(z)\right|, \\
& S_{2}=\limsup _{n \longrightarrow \infty} \sup _{|\psi(z)| \leq \delta_{N}} \omega(z)\left|\xi(z)\left\|\left(f-f_{\delta_{n}}\right)^{\prime}(\psi(z))\right\| \psi^{\prime}(z)\right|, \\
& S_{3}=\limsup _{n \longrightarrow \infty} \sup _{|\psi(z)| \leq \delta_{N}} \omega(z)\left|\xi^{\prime}(z)\left\|\left(f-f_{\delta_{n}}\right)(\psi(z))\right\|\right|, \\
& S_{4}=\limsup _{n \longrightarrow \infty} \sup _{|\psi(z)| \leq \delta_{N}} \omega(z)\left|\xi^{\prime}(z)\left\|\left(f-f_{\delta_{n}}\right)(\psi(z))\right\|\right|
\end{aligned}
$$

Taking the operator $\mathscr{W}_{\xi, \psi}$ to 1 and $z$ and using its boundedness, it easily follows that $\xi \in \mathbb{B}_{\omega}$ and

$$
\widetilde{E}=\sup _{z \in \mathbb{D}} \omega(z)\left|\psi^{\prime}(z) \xi(z)\right|<\infty .
$$

Also, on compact subsets of $\mathbb{D}, \delta_{n} f_{\delta_{n}}{ }^{\prime}$ uniformly converges to $f^{\prime}$ as $n \longrightarrow \infty$; thus, we have

$$
S_{1} \leq \widetilde{E} \limsup \sup _{n \longrightarrow \infty}\left|f^{\prime}(w)-\delta_{N} \delta_{n} f^{\prime}\left(\delta_{n} w\right)\right|=0 .
$$

Similarly, for $\xi \in \mathbb{B}_{\omega}$ and the fact that $f_{\delta_{n}}$ converges uniformly to $f$ on compact subsets of $\mathbb{D}$ as $n \longrightarrow \infty$, we obtain

$$
S_{3} \leq\|\xi\|_{\mathfrak{B}_{\omega}} \limsup _{n \longrightarrow \infty} \sup _{w \leq \delta_{N}}\left|f(w)-f\left(\delta_{n} w\right)\right|=0 .
$$

Now, consider $S_{2}$. We have $S_{2} \leq \lim \sup _{n \longrightarrow \infty}\left(P_{1}+P_{2}\right)$, where

$$
\begin{aligned}
& P_{1}=\sup _{|\psi(z)|>\delta_{N}} \omega(z)\left|\xi(z)\left\|\psi^{\prime}(z)\right\| f^{\prime}(\psi(z))\right|, \\
& P_{2}=\sup _{|\psi(z)|>\delta_{N}} \delta_{n} \omega(z)\left|\xi(z)\left\|\psi^{\prime}(z)\right\| f^{\prime}\left(\delta_{n} \psi(z)\right)\right| .
\end{aligned}
$$

First, we consider $P_{1}$. As $\|f\|_{\mathfrak{D}_{K}} \leq 1$, we obtain

$$
\begin{aligned}
P_{1} & =\sup _{|\psi(z)|>\delta_{N}} \omega(z)\left|\xi(z)\left\|\psi^{\prime}(z)\right\| f^{\prime}(\psi(z))\right| \\
& \leq \frac{1}{\delta_{N}}\|f\|_{\mathfrak{D}_{K}} \sup _{|\psi(z)|>\delta_{N}} \omega(z)\left|\xi(z) \| \psi^{\prime}(z)\right| \frac{|\psi(z)|}{\sqrt{K\left(1-|\psi(z)|^{2}\right)\left(1-|\psi(z)|^{2}\right)^{2}}}
\end{aligned}
$$

On taking limit as $N \longrightarrow \infty$, we obtain

$$
\limsup _{n \rightarrow \infty} P_{1} \leq \limsup _{|\psi(z)| \longrightarrow 1} \frac{\omega(z)\left|\xi(z) \psi^{\prime}(z)\right|}{\sqrt{K\left(1-|\psi(z)|^{2}\right)}\left(1-|\psi(z)|^{2}\right)^{2}}=S .
$$

In the similar manner, we obtain

$$
\limsup _{n \rightarrow \infty} P_{2} \leq \limsup _{|\psi(z)| \longrightarrow 1} \frac{\omega(z)\left|\xi(z) \psi^{\prime}(z)\right|}{\sqrt{K\left(1-|\psi(z)|^{2}\right)}\left(1-|\psi(z)|^{2}\right)^{2}}=S .
$$

On combining the above two inequalities, we obtain

$$
S_{2} \leq \limsup _{n \longrightarrow \infty}\left(P_{1}+P_{2}\right) \leq S .
$$
where

Next, consider $S_{4}$. We have $S_{4} \leq \lim \sup _{n \longrightarrow \infty}\left(P_{3}+P_{4}\right)$,

$$
\begin{aligned}
& P_{3}=\sup _{|\psi(z)|>\delta_{N}} \omega(z)\left|\xi^{\prime}(z) \| f(\psi(z))\right|, \\
& P_{4}=\sup _{|\psi(z)|>\delta_{N}} \omega(z)\left|\xi^{\prime}(z) \| f\left(\delta_{n} \psi(z)\right)\right| .
\end{aligned}
$$

By similar calculation, we obtain

$$
\begin{aligned}
P_{3} & =\sup _{|\psi(z)|>\delta_{N}} \omega(z)\left|\xi^{\prime}(z) \| f(\psi(z))\right| \\
& \leq \frac{1}{\delta_{N}}\|f\|_{\mathfrak{D}_{K}} \sup _{|\psi(z)|>\delta_{N}} \omega(z)\left|\xi^{\prime}(z)\right| \frac{1}{\sqrt{K\left(1-|\psi(z)|^{2}\right)}\left(1-|\psi(z)|^{2}\right)}
\end{aligned}
$$

On taking limit $N \longrightarrow \infty$, we obtain 
$\limsup _{n \rightarrow \infty} P_{3} \lesssim \limsup _{|\psi(z)| \longrightarrow 1} \frac{\omega(z)\left|\xi^{\prime}(z)\right|}{\sqrt{K\left(1-|\psi(z)|^{2}\right)}\left(1-|\psi(z)|^{2}\right)}=R$.
In the similar manner, we obtain

$$
\limsup _{n \longrightarrow \infty} P_{4} \lesssim \limsup _{|\psi(z)| \longrightarrow 1} \frac{\omega(z)\left|\xi^{\prime}(z)\right|}{\sqrt{K\left(1-|\psi(z)|^{2}\right)}\left(1-|\psi(z)|^{2}\right)}=R .
$$

Combining the above two inequalities, we obtain

$$
S_{4} \leq \limsup _{n \longrightarrow \infty}\left(P_{3}+P_{4}\right) \leq R
$$

On combining (40), (43), (44), (49), and (54), we obtain

$$
\begin{aligned}
\limsup _{n \longrightarrow \infty}\left\|\mathscr{W}_{\xi, \psi}-\mathscr{W}_{\xi, \psi} E_{\delta_{n}}\right\|_{\mathfrak{D}_{K} \longrightarrow \mathfrak{B}_{\omega}} & =\limsup _{n \longrightarrow \infty} \sup _{\|f\|_{\mathfrak{D}_{K}} \leq 1}\left\|\left(\mathscr{W}_{\xi, \psi}-\mathscr{W}_{\xi, \psi} E_{\delta_{n}}\right) f\right\|_{\mathfrak{B}_{\omega}} \\
& =\limsup _{n \longrightarrow \infty} \sup _{\|f\|_{\mathfrak{D}_{K}} \leq 1}\left\|\xi\left(f-f_{\delta_{n}}\right) \circ \psi\right\|_{\mathfrak{B}_{\omega}} \\
& \leq R+S .
\end{aligned}
$$

Thus, inequalities (37) and (55) imply that

$$
\left\|\mathscr{W}_{\xi, \psi}\right\|_{e, \mathfrak{D}_{K} \longrightarrow \mathfrak{B}_{\omega}} \leq R+S \lesssim \max \{R, S\} .
$$

Hence, inequalities (34) and (56) complete the theorem.

The following corollary can be easily obtained from Theorem 5.

Corollary 1. Let $\omega$ be a standard weight and $\psi$ be a selfanalytic map on $\mathbb{D}$. Let $K$ be a weight function. Assume that $C_{\psi}: \mathfrak{D}_{K} \longrightarrow \mathfrak{B}_{\omega}$ is bounded. Then,

$$
\left\|C_{\psi}\right\|_{e, \mathfrak{D}_{K} \longrightarrow \mathfrak{B}_{\omega}} \approx \limsup _{|\psi(z)| \longrightarrow 1} \frac{\omega(z)\left|\psi^{\prime}(z)\right|}{\sqrt{K\left(1-|\psi(z)|^{2}\right)}\left(1-|\psi(z)|^{2}\right)^{2}} .
$$

\section{Essential Norm of Weighted Composition Operator from Dirichlet-Type Space to Bers-Type Space}

In this section, we consider the Bers-type spaces and estimated the essential norm of weighted composition operator from $\mathfrak{D}_{K}$ to $\mathfrak{\mathfrak { A }}_{\omega}$.

Theorem 6. Let $\omega$ be a standard weight, $\xi \in H(\mathbb{D})$, and $\psi$ be a self-analytic map on $\mathbb{D}$. Let $K$ be a weight function. Assume that the operator $\mathscr{W}_{\xi, \psi}: \mathfrak{D}_{K} \longrightarrow \mathfrak{A}_{\omega}$ is bounded. Then,

$$
\left\|\mathscr{W}_{\xi, \psi}\right\|_{e, \mathfrak{D}_{K} \longrightarrow \mathfrak{A}_{\omega}} \approx \limsup _{|\psi(z)| \longrightarrow 1^{-}} \frac{\omega(z)|\xi(z)|}{\sqrt{K\left(1-|\psi(z)|^{2}\right)}\left(1-|\psi(z)|^{2}\right)} .
$$

Proof. Firstly, we prove that

$$
\left\|\mathscr{W}_{\xi, \psi}\right\|_{e, \mathfrak{D}_{K} \longrightarrow \mathfrak{A}_{\omega}} \geq \limsup _{|\psi(z)| \longrightarrow 1^{-}} \frac{\omega(z)|\xi(z)|}{\sqrt{K\left(1-|\psi(z)|^{2}\right)}\left(1-|\psi(z)|^{2}\right)} .
$$

Consider a function $f_{z} \in \mathfrak{D}_{K}$ such that $\left\|f_{z}\right\|_{\mathfrak{D}_{K}} \lesssim 1$, and on compact subsets of $\mathbb{D}, f_{z}$ converges to zero as $|z| \longrightarrow 1$. Thus, for any compact operator $E: \mathfrak{D}_{K} \longrightarrow \mathfrak{A}_{\omega}$ and any $\left(\zeta_{n}\right)_{n \in \mathbb{D}}$ such that $\left|\psi\left(\zeta_{n}\right)\right| \longrightarrow 1^{-}$, we obtain

$$
\begin{aligned}
\left\|\mathscr{W}_{\xi, \psi}-E\right\|_{\mathfrak{D}_{K} \longrightarrow \mathfrak{A}_{\omega}} & \gtrless\left\|\left(\mathscr{W}_{\xi, \psi}-E\right) f_{\psi\left(\zeta_{n}\right)}\right\|_{\mathfrak{A}_{\omega}} \\
& \geq\left\|\mathscr{W}_{\xi, \psi} f_{\psi}\left(\zeta_{n}\right)\right\|_{\mathfrak{B}_{\omega}}-\left\|E f_{\psi\left(\zeta_{n}\right)}\right\|_{\mathfrak{B}_{\omega}} .
\end{aligned}
$$

Taking $\lim \sup _{\left|\psi\left(\zeta_{n} a\right)\right|} \longrightarrow 1^{-}$on both sides, we obtain

$$
\begin{aligned}
\left\|\mathscr{W}_{\xi, \psi}-E\right\|_{\mathfrak{D}_{K} \longrightarrow \mathfrak{A}_{\omega}} & \gtrsim \limsup _{\left|\psi\left(\zeta_{n}\right)\right| \longrightarrow 1^{-}}\left\|\mathscr{W}_{\xi, \psi} f_{\psi\left(\zeta_{n}\right)}\right\|_{\mathfrak{A}_{\omega}} \\
& \gtrsim \limsup _{\left|\psi\left(\zeta_{n}\right)\right| \longrightarrow 1^{-}} \frac{\omega\left(\zeta_{n}\right)\left|\xi\left(\zeta_{n}\right)\right|}{\sqrt{K\left(1-\left|\psi\left(\zeta_{n}\right)\right|^{2}\right)}\left(1-\left|\psi\left(\zeta_{n}\right)\right|^{2}\right)}
\end{aligned}
$$

On applying the definition of essential norm, we find that 


$$
\left\|\mathscr{W}_{\xi, \psi}\right\|_{e, \mathfrak{D}_{K} \longrightarrow \mathfrak{A}_{\omega}}=\inf _{E}\left\|\mathscr{W}_{\xi, \psi}-E\right\|_{\mathcal{D}_{K} \rightarrow \mathfrak{A}_{\omega}} \approx \limsup _{\left|\psi\left(\zeta_{n}\right)\right| \rightarrow 1^{-}} \frac{\omega\left(\zeta_{n}\right)\left|\xi\left(\zeta_{n}\right)\right|}{\sqrt{K\left(1-\left|\psi\left(\zeta_{n}\right)\right|^{2}\right)\left(1-\left|\psi\left(\zeta_{n}\right)\right|^{2}\right)}}
$$

Finally, we prove that

$$
\left\|\mathscr{W}_{\xi, \psi}\right\|_{e, D_{K} \rightarrow \mathfrak{A}_{\omega}} \leqslant \limsup _{\left|\psi\left(\zeta_{n}\right)\right| \rightarrow 1^{-}} \frac{\omega\left(\zeta_{n}\right)\left|\xi\left(\zeta_{n}\right)\right|}{\sqrt{K\left(1-\left|\psi\left(\zeta_{n}\right)\right|^{2}\right)}\left(1-\left|\psi\left(\zeta_{n}\right)\right|^{2}\right)} .
$$

For this, consider $E_{\delta}: H(\mathbb{D}) \longrightarrow H(\mathbb{D})$ with $\delta \in[0,1)$ and a sequence $\left\{\delta_{n}\right\} \subset(0,1)$ satisfying $\delta_{n} \longrightarrow 1$ as $n \longrightarrow \infty$ defined in Theorem 5. Then, for all $n \in \mathbb{N}$, the operator $\mathscr{W}_{\xi, \psi} E_{\delta_{n}}: \mathfrak{D}_{K} \longrightarrow \mathfrak{A}_{\omega}$ is compact. By using the definition of essential norm, we obtain

$$
\left\|\mathscr{W}_{\xi, \psi}\right\|_{e, \mathfrak{D}_{K} \longrightarrow \mathfrak{A}_{\omega}} \leq \limsup _{n \longrightarrow \infty}\left\|\mathscr{W}_{\xi, \psi}-\mathscr{W}_{\xi, \psi} E_{\delta_{n}}\right\|_{\mathcal{D}_{K} \longrightarrow \mathfrak{A}_{\omega}} .
$$

So, we only have to prove that

$$
\begin{aligned}
& \limsup _{n \longrightarrow \infty}\left\|\mathscr{W}_{\xi, \psi}-\mathscr{W}_{\xi, \psi} E_{\delta_{n}}\right\|_{\mathfrak{D}_{K} \rightarrow \mathfrak{B}_{\omega}} \\
& \quad \leqslant \limsup _{\left|\psi\left(\zeta_{n}\right)\right| \longrightarrow 1^{-}} \frac{\omega\left(\zeta_{n}\right)\left|\xi\left(\zeta_{n}\right)\right|}{\sqrt{K\left(1-\left|\psi\left(\zeta_{n}\right)\right|^{2}\right)}\left(1-\left|\psi\left(\zeta_{n}\right)\right|^{2}\right)}
\end{aligned}
$$
have

Let $f$ be a function in $\mathfrak{D}_{K}$ satisfying $\|f\|_{\mathfrak{D}_{K}} \leq 1$; then, we

$$
\left\|\left(\mathscr{W}_{\xi, \psi}-\mathscr{W}_{\xi, \psi} E_{\delta_{n}}\right) f\right\|_{\mathcal{D}_{K} \longrightarrow \mathfrak{A}_{\omega}}=\left\|\xi\left(f-f_{\delta_{n}}\right) \circ \psi\right\|_{\mathfrak{A}_{\omega}} .
$$

Furthermore, consider a large enough $N \in \mathbb{N}$ such that, for all $n \geq N$, we have $\delta_{n} \geq 1 / 2$. Thus, we obtain where

$$
\begin{aligned}
& A_{1}=\limsup _{n \longrightarrow \infty} \sup _{|\psi(z)| \leq \delta_{N}} \omega(z)|\xi(z)|\left(f-f_{\delta_{n}}\right)(\psi(z)) \mid, \\
& A_{2}=\limsup _{n \longrightarrow \infty} \sup _{|\psi(z)| \leq \delta_{N}} \omega(z)|\xi(z)|\left(f-f_{\delta_{n}}\right)(\psi(z)) \mid .
\end{aligned}
$$

Similar to Theorem 5 , for $\xi \in \mathfrak{A}_{\omega}$ and the fact that $f_{\delta_{n}}$ converges uniformly to $f$ on compact subsets of $\mathbb{D}$ as $n \longrightarrow \infty$, we obtain

$$
A_{1} \leq\|\xi\|_{\mathscr{A}_{\omega}} \limsup _{n \longrightarrow \infty} \sup _{w \leq \delta_{N}}\left|f(w)-f\left(\delta_{n} w\right)\right|=0 .
$$

Next, we consider $A_{2}$. We have $A_{2} \leq$ $\lim \sup _{n \rightarrow \infty}\left(R_{1}+R_{2}\right)$, where

$$
\begin{aligned}
& R_{1}=\sup _{|\psi(z)|>\delta_{N}} \omega(z)|\xi(z) \| f(\psi(z))|, \\
& R_{2}=\sup _{|\psi(z)|>\delta_{N}} \omega(z)\left|\xi(z) \| f\left(\delta_{n} \psi(z)\right)\right| .
\end{aligned}
$$

On calculation, we obtain
Similarly,

$$
\limsup _{n \longrightarrow \infty} R_{2} \lesssim \limsup _{|\psi(z)| \longrightarrow 1^{-}} \frac{\omega(z)|\xi(z)|}{\sqrt{K\left(1-|\psi(z)|^{2}\right)}\left(1-|\psi(z)|^{2}\right)} \text {. }
$$

On combining the above two inequalities, we obtain

$$
A_{2} \leq \limsup _{n \longrightarrow \infty}\left(R_{1}+R_{2}\right) \lesssim \limsup _{|\psi(z)| \longrightarrow 1^{-}} \frac{\omega(z)|\xi(z)|}{\sqrt{K\left(1-|\psi(z)|^{2}\right)}\left(1-|\psi(z)|^{2}\right)} .
$$

From inequalities (67), (69), and (74), we obtain 


$$
\begin{aligned}
\limsup _{n \longrightarrow \infty}\left\|\mathscr{W}_{\xi, \psi}-\mathscr{W}_{\xi, \psi} E_{\delta_{n}}\right\|_{\mathfrak{D}_{K} \rightarrow \mathfrak{A}_{\omega}} & =\limsup _{n \longrightarrow \infty} \sup _{\|f\|_{\mathfrak{D}_{K} \leq 1}}\left\|\left(\mathscr{W}_{\xi, \psi}-\mathscr{W}_{\xi, \psi} E_{\delta_{n}}\right) f\right\|_{\mathfrak{A}_{\omega}} \\
& =\limsup _{n \longrightarrow \infty} \sup _{\|f\|_{\mathfrak{D}_{K} \leq 1}}\left\|\xi\left(f-f_{\delta_{n}}\right) \circ \psi\right\|_{\mathfrak{A}_{\omega}} \\
& \lesssim \limsup _{|\psi(z)| \longrightarrow 1^{-}} \frac{\omega(z)|\xi(z)|}{\sqrt{K\left(1-|\psi(z)|^{2}\right)}\left(1-|\psi(z)|^{2}\right)}
\end{aligned}
$$

Thus, inequalities (64) and (75) imply that

$$
\left\|\mathscr{W}_{\xi, \psi}\right\|_{e, \mathfrak{D}_{K} \longrightarrow \mathfrak{A}_{\omega}} \lesssim \limsup _{|\psi(z)| \longrightarrow 1^{-}} \frac{\omega(z)|\xi(z)|}{\sqrt{K\left(1-|\psi(z)|^{2}\right)}\left(1-|\psi(z)|^{2}\right)} .
$$

Hence, inequalities (62) and (76) complete the theorem. The following corollary can be easily obtained from Theorem 6.

Corollary 2. Let $\omega$ be a standard weight and $\psi$ be a selfanalytic map on $\mathbb{D}$. Let $K$ be a weight function. Assume that the operator $C_{\psi}: \mathfrak{D}_{K} \longrightarrow \mathfrak{A}_{\omega}$ is bounded. Then,

$$
\left\|C_{\psi}\right\|_{e, \mathfrak{D}_{K} \longrightarrow \mathfrak{A}_{\omega}} \approx \limsup _{|\psi(z)| \longrightarrow 1^{-}} \frac{\omega(z)}{\sqrt{K\left(1-|\psi(z)|^{2}\right)}\left(1-|\psi(z)|^{2}\right)} .
$$

\section{Data Availability}

No data were used to support this study.

\section{Conflicts of Interest}

The authors declare that they have no conflicts of interest.

\section{References}

[1] R. F. Allen and F. Colonna, "Weighted composition operators on the Bloch space of a bounded homogeneous domain," Topics in Operator Theory, vol. 202, pp. 11-37, 2010.

[2] F. Colonna and S. Li, "Weighted composition operators from the minimal möbius invariant space into the bloch space," Mediterranean Journal of Mathematics, vol. 10, pp. 395-409, 2013.

[3] M. Devi, A. K. Sharma, and K. Raj, "Weighted composition operators from Dirichlet type spaces to some weighted-type spaces," Journal of Computational Analysis and Applications, vol. 28, pp. 127-135, 2020.

[4] Z. J. Jiang, "Weighted composition operators from weighted Bergman spaces to some spaces of analytic functions on the upper half plane," Utliltas Mathematica, vol. 93, pp. 205-212, 2014.

[5] S. Li and S. Stević, "Weighted composition operators from bergman-type spaces into bloch spaces," Proceedings Mathematical Sciences, vol. 117, no. 3, pp. 371-385, 2007.

[6] S. Li and S. Stević, "Weighted composition operators from $H^{\infty}$ to the bloch space on the polydisc," Abstract and Applied Analysis, vol. 2007, Article ID 048478, 13 pages, 2007.
[7] S. Li and S. Stević, "Weighted composition operators from zygmund spaces into bloch spaces," Applied Mathematics and Computation, vol. 206, no. 2, pp. 825-831, 2008.

[8] S. Li and S. Stevi'c, "Weighted composition operators between $\mathrm{H} \infty$ and $\alpha$-BLOCH spaces IN the unit ball," Taiwanese Journal of Mathematics, vol. 12, no. 7, pp. 1625-1639, 2008.

[9] R. Rochberg and Z. Wu, "A new characterization of Dirichlet type spaces and applications," Illinois Journal of Mathematics, vol. 37, no. 1, pp. 101-122, 1993.

[10] A. K. Sharma and S. I. Ueki, "Compact composition operator on the Bloch space and the growth space of the upper halfplane," Mediterranean Journal of Mathematics, vol. 14, no. 2, p. 9, 2017.

[11] S. Stević, A. K. Sharma, and S. D. Sharma, "Generalized integration operators from the space of integral transforms into Bloch type spaces," Journal of Computational Analysis and Applications, vol. 14, pp. 1139-1147, 2012.

[12] S. Stević, "Weighted composition operators from weighted Bergman spaces to weighted-type spaces on the unit ball," Applied Mathematics and Computation, vol. 212, pp. 499-504, 2009.

[13] S. Stević and R. P. Agarwal, "Weighted composition operators from logarithmic Bloch-type spaces to Bloch-type spaces," Journal of Inequalities and Applications, vol. 2009, Article ID 964814, 2009.

[14] W. Yang, "Weighted composition operators from Bloch-type spaces to weighted-type spaces," Ars Combinatoria, vol. 93, pp. 265-274, 2009.

[15] C. C. Cowen and B. D. MacCluer, Composition Operators on Spaces of Analytic Functions, CRC Press, Boca Raton, FL, USA, 1995.

[16] K. Zhu, Spaces of Holomorphic Functions in the Unit Ball, Springer, New York, NY, USA, 2005.

[17] A. Aleman, "Hilbert spaces of analytic functions between the hardy and the dirichlet space," Proceedings of the American Mathematical Society, vol. 115, no. 1, p. 97, 1992.

[18] R. Kerman and E. Sawyer, "Carleson measures and multipliers of Dirichlet-type spaces," Transactions of the American Mathematical Society, vol. 309, no. 1, p. 87, 1988.

[19] H. Wulan and K. Zhu, "Lacunary series in QKspaces," Studia Mathematica, vol. 178, no. 3, pp. 217-230, 2007.

[20] H. Wulan and J. Zhou, "Q_K and Morrey type spaces," Annales Academiae Scientiarum Fennicae Mathematica, vol. 38, pp. 193-207, 2013.

[21] G. Bao, Z. Lou, R. Qian, and H. Wulan, "On multipliers of Dirichlet type spaces," Complex Analysis and Operator Theory, vol. 9, no. 8, pp. 1701-1732, 2015.

[22] H. A. Alsaker, Multipliers of the Dirichlet Space, Master's thesis, The University of Bergen, Bergen, Norway, 2009.

[23] N. Arcozzi, R. Rochberg, E. T. Sawyer, and B. D. Wick, "The Dirichlet space: a survey," New York Journal of Mathematics, vol. 17A, pp. 45-86, 2011. 
[24] D. A. Stegenga, "Multipliers of the dirichlet space," Illinois Journal of Mathematics, vol. 24, no. 1, pp. 113-139, 1980.

[25] G. D. Taylor, "Multipliers on $\$ D \backslash$ sb $\{\backslash$ alpha $\} \$$," Transactions of the American Mathematical Society, vol. 123, no. 1, p. 229, 1966.

[26] M. Essen, H. Wulan, and J. Xiao, "Several function-theoretic characterizations of Möbius invariant $\mathbb{Q}_{K}$ spaces," Journal of Functional Analysis, vol. 230, pp. 78-115, 2006.

[27] F. Gürbüz, "Some estimates for generalized commutators of rough fractional maximal and integral operators on generalized weighted morrey spaces," Canadian Mathematical Bulletin, vol. 60, no. 1, pp. 131-145, 2017.

[28] F. Gürbüz, "Generalized weighted Morrey estimates for Marcinkiewicz integrals with rough kernel associated with schrödinger operator and their commutators," Chinese Annals of Mathematics, Series B, vol. 41, no. 1, pp. 77-98, 2020.

[29] F. Gürbüz, "On the behaviors of rough multilinear fractional integral and multi-sublinear fractional maximal operators both on product Lp and weighted Lp spaces," International Journal of Nonlinear Sciences and Numerical Simulation, vol. 21, no. 7-8, pp. 715-726, 2020.

[30] F. Gürbüz, "Some inequalities for the multilinear singular integrals with Lipschitz functions on weighted Morrey spaces," Journal of Inequalities and Applications, vol. 2020, 2020.

[31] S. Li, R. Qian, and J. Zhou, "Essential norm and a new characterization of weighted composition operators from weighted Bergman spaces and Hardy spaces into the Bloch space," Czechoslovak Mathematical Journal, vol. 67, no. 3, pp. 629-643, 2017.

[32] Y.-X. Liang and Z.-H. Zhou, "The products of differentiation and composition operators from logarithmic Bloch spaces to \$\$ \mu \$ \$-Bloch spaces," Bulletin of the Iranian Mathematical Society, vol. 46, no. 1, pp. 159-176, 2020.

[33] B. D. MacCluer and R. Zhao, "Essential norms of weighted composition operators between bloch-type spaces," Rocky Mountain Journal of Mathematics, vol. 33, no. 4, pp. 14371458, 2003.

[34] J. S. Manhas and R. Zhao, "New estimates of essential norms of weighted composition operators between bloch type spaces," Journal of Mathematical Analysis and Applications, vol. 389, no. 1, pp. 32-47, 2012.

[35] S. Stević, "Essential norms of weighted composition operators from the $\alpha$-Bloch space to a weighted-type space on the unit ball," Abstract and Applied Analysis, vol. 2008, Article ID 279691, 11 pages, 2008.

[36] Y. Yu and Y. Liu, "The essential norm of a generalized composition operator between bloch-type spaces and Q K type spaces," Complex Analysis and Operator Theory, vol. 6, no. 6, pp. 1231-1240, 2012.

[37] H.-G. Zeng and Z.-H. Zhou, "Essential norm estimate of a composition operator between Bloch-type spaces in the unit ball," Rocky Mountain Journal of Mathematics, vol. 42, no. 3, pp. 1049-1071, 2012. 